\title{
Chronic prostatitis effectively managed by transurethral prostatectomy (TURP) in a spinal cord injury male
}

\author{
Charalampos Konstantinidis (10 ${ }^{1} \cdot$ Achileas Karafotias $^{2} \cdot$ Ioannis Eleftheropoulos $^{2} \cdot$ Dimitrios Delakas $^{2}$
}

Received: 19 May 2019 / Revised: 28 August 2019 / Accepted: 1 September 2019

(c) International Spinal Cord Society 2019

\begin{abstract}
Introduction Spinal cord injury (SCI), specifically suprasacral SCI, results in high intravesical pressures, elevated post-void residual and urinary incontinence which are all risk factors for urinary tract infections (UTIs). The management of UTIs usually is conservative medical antibiotic treatment. However, recurrent UTIs in the SCI patient population warrant further investigation. The method of urinary drainage (intermittent or indwelling urinary catheters, urinary diversion) and untreated complications of NLUTD (vesicoureteral reflux, stone formation, chronic incomplete emptying of the bladder) are risk factors for recurrent UTIs (rUTIs). Removal of these UTI risk factors and improving urinary drainage are goals of urologic management; however, when conservative interventions do not succeed, surgery may be a viable solution in select cases of rUTIs.

Case presentation We present a case of complicated persisting rUTIs and associated urethral discharge in a middle-aged SCI male who manages his bladder with intermittent catheterization (IC). We detail the evaluation and management approach that leads to an eventual transurethral prostatectomy (TURP) as a final solution for his rUTIs. Fortunately, the surgical intervention was successful, and the patient is free of UTIs after 4 years of follow-up.

Discussion In SCI male patients with rUTIs and suspected chronic prostatitis, TURP may be a valuable treatment option once all predisposing factors have been remediated.
\end{abstract}

\section{Introduction}

Neurogenic lower urinary tract (LUT) dysfunction can be caused by injuries of the spinal cord (SCI). High intravesical pressure, post-void residual and incontinence are the main consequences of this dysfunction and all of these conditions can cause urinary tract infections (UTIs). In addition, the potential complications of neurogenic urinary disorders (reflux, stone formation, incomplete emptying of the bladder), and the methods of urine drainage (intermittent or indwelling catheters, urinary diversion), contribute even more to UTIs. In persons with SCIs, all UTIs are considered

Charalampos Konstantinidis

konstantinidischaralampos@yahoo.com

1 Urology \& Neuro-urology Unit, National Rehabilitation Center, Athens, Greece

2 Urology Department, General hospital “Asklepieio Voulas”, Athens, Greece as complicated ones and there is different microbiology as compared to the general population [1-3].

Recurrent infections of the urinary tract (rUTI) usually are treated conservatively. When these methods fail, surgical treatment is an option. In order to proceed in surgery, a constant source of microorganisms and/or appropriate conditions for their proliferation should be present. Sources of infections include calculi, fistulae or abscesses. Favorable conditions for bacterial proliferation can occur under malignancies, foreign bodies or high post-void residual volumes [4].

There are four clinical types of prostatitis: acute bacterial prostatitis, chronic bacterial prostatitis, chronic nonbacterial prostatitis/pelvic pain syndrome, and asymptomatic inflammatory prostatitis [3]. Common treatment means are antibiotics, alpha-blockers, hormonal treatment, antiinflammatory drugs and phytotherapeutic agents (plant extracts) [5]. Transurethral prostatectomy (TURP) is suggested as a potential treatment option when other therapeutic approaches fail, but it is not widely used as such $[6,7]$.

This report analyses the sequence of diagnosis and treatment for a case of complicated rUTIs in a middle-aged 
man with paraplegia and neurogenic LUT dysfunction. The final therapeutic step for the patient was a TURP, which resulted in the patient being free from rUTIs at his 4-year follow-up.

\section{Case presentation}

This case involves a 52-year-old male with T12 AIS A SCI secondary to a car accident in 2012 [8, 9]. His bladder management consisted of intermittent catheterization (IC) 4-5 times per day in addition to using a condom catheter for concomitant urinary incontinence, especially during his wheelchair basketball sports activity. The patient had a 6-year history of 3-4 Escherichia coli rUTIs per year accompanied by high fever with chills and associated temporary urethral secretion. With the onset of the UTI symptoms, his urinary incontinence ceased temporarily and the following day he would notice a thick, purulent urethral discharge. Repeated renal ultrasound evaluation did not reveal stones, renal dilatation or parenchymal abnormalities.

Due to the urethral discharge, a periurethral source of infection/abscess was suspected. A urethral swab/secretion culture revealed $>10,000 E$. coli $\mathrm{cfu} / \mathrm{hpf}$. Transrectal prostate ultrasound (TRUS) and lower abdominal magnetic resonance imaging (MRI) were conducted and both tests were without any significant findings.

Urodynamics in the absence of UTI demonstrated a stable bladder pressure system with neurogenic detrusor underactivity accompanied by stress urinary incontinence due to urethral sphincter deficiency. Bladder compliance was normal at 79 .

Cystourethroscopy demonstrated a normal urethra and bladder; however, there was an incidental hypertrophic verum montanum (Fig. 1). Given the limited findings yet rUTIs, it was hypothesized that the hypertrophic verum

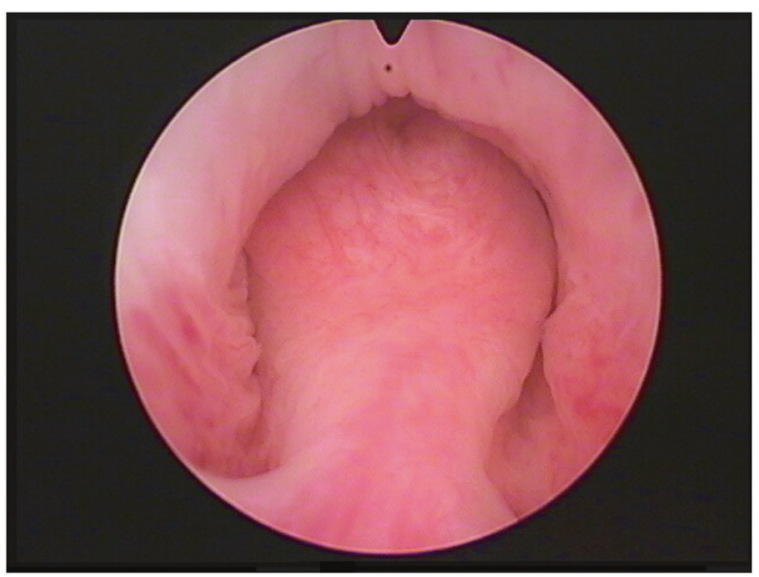

Fig. 1 Urethroscopic view of the hypertrophic verum montanum

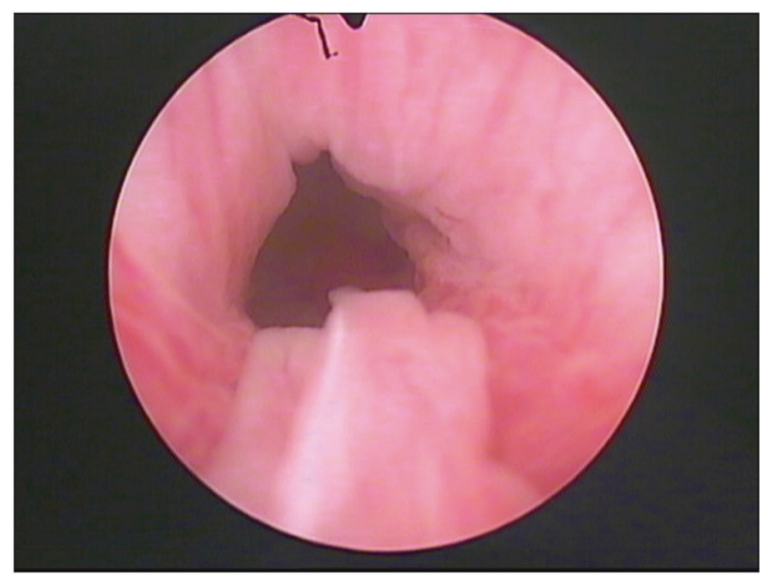

Fig. 2 Urethroscopic view of the resected verum montanum

montanum was acting as a source of temporary obstruction during a prostatitis inflammation process. After thorough discussion, the individual underwent endoscopic removal of verum montanum (Fig. 2).

Urodynamic studies were repeated post verum montanum resection demonstrating similar neurogenic detrusor underactivity and urethral sphincter deficiency with an abdominal leak point pressure (ALPP) at $100 \mathrm{cmH}_{2} \mathrm{O}$. Clinical recommendations for management consisted of continuing self-IC 4-5 times per day and avoidance of Valsalva/straining maneuvers for bladder emptying. Our goal to avoid rUTIs was to maintain a low-pressure reservoir that was emptied completely via IC and to avoid Valsalva/strain voiding to minimize pressure in the prostatic area.

Due to the failure of appropriate culture-sensitive antibiotics to eradicate the rUTIs and the new findings of prostatic calcifications (Fig. 3), a TURP was recommended. An uneventful TURP was performed 3 months after the last rUTI, and pathology revealed chronic inflammatory elements and small abscesses in the prostate gland (Fig. 4). The individual has been followed for the past 4 years and he has been free of UTI symptoms and urethral discharge after the TURP.

Urodynamic testing was repeated 1 year after the TURP and demonstrated stable bladder pressures with neurogenic detrusor underactivity and stress urinary incontinence with urethral sphincter deficiency but now with a lower ALPP of $70 \mathrm{cmH}_{2} \mathrm{O}$. The lower ALPP can be explained by the decreasing of "urethral resistance" after TURP. The individual continues to manage his bladder by IC and avoid straining. To manage his stress incontinence due to sphincter deficiency, he continues to use a condom catheter. The TURP did not have any adverse effects on his bowel or sexual function. The individual also uses intracavernosal alprostadil injections for his neurogenic erectile dysfunction with good results. 

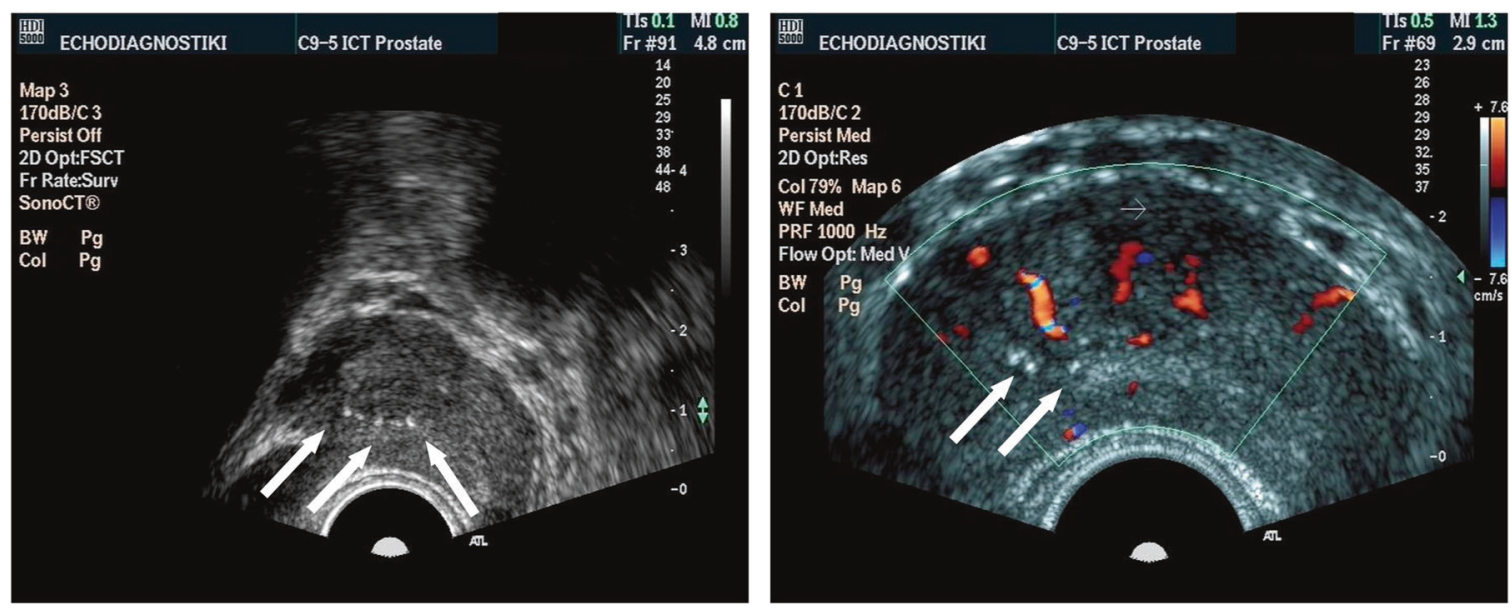

Fig. 3 Prostatic calcifications in TRUS of the prostate
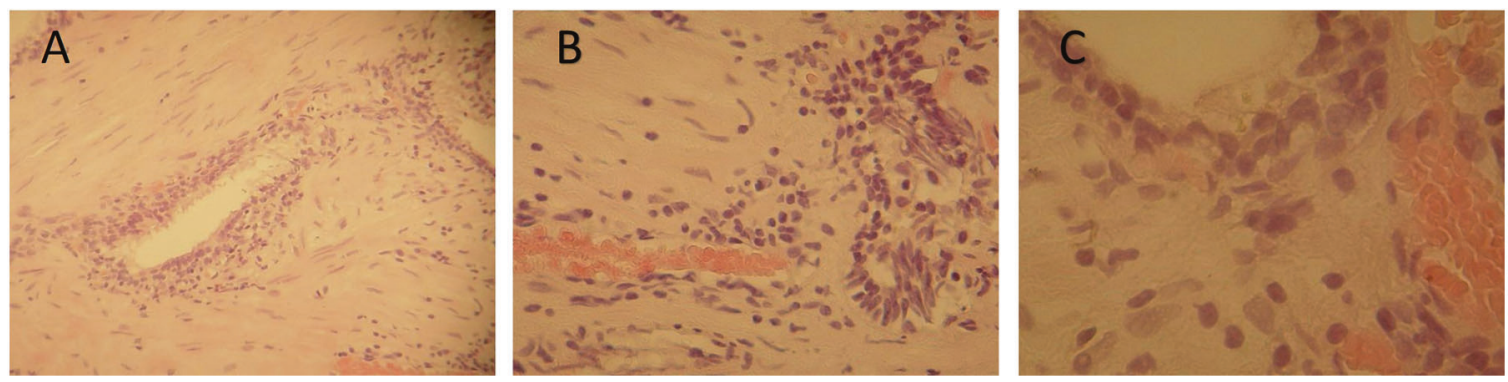

Fig. 4 Hematoxylin/eosin-stained prostate tissue. Numerous small dark blue lymphocytes are seen in the stroma between the glands as of chronic prostate inflammation. Zoom $\times 100($ a),$\times 200($ (b) and $\times 400(\mathbf{c})$

\section{Discussion}

To our knowledge, this is the first reported case of successful TURP treatment of chronic prostatitis in an individual with SCI. TURP has been reported as a therapeutic approach to prostatitis in six case studies from 1973 to 2014 [10]. One-hundred and ten men were included in the series and none of them had SCIs. In the series, the mean age at diagnosis was 59 years (39-81 years) and symptom duration ranged from 6 to 30 months, for 82 patients. Prior to TURP, patients were treated with conservative antibiotic therapy and alpha-blockers. Out of 110 patients, 78 (70\%) were referred as cured, $16(15 \%)$ as improved and $16(15 \%)$ with no change. Only one patient's symptoms were quantified under the International Prostate Symptom Score (IPSS), in which improvement suggested, with a score from 27 downgraded to 3 postoperatively [2].

Chronic bacterial prostatitis is a relatively rare male condition that presents with vague recurrent UTI symptoms. The prevalence of prostatitis-like symptoms is only $8 \%$. Due to the high relapse rate, it can have a significant negative impact on a man's quality of life. While the treatment of acute bacterial prostatitis should definitely rely on antibiotics, there are no clear recommendations for the management of chronic prostatitis.

Our case report documents a role for surgical intervention when conservative medical therapy for chronic prostatitis is ineffective. Surgical TURP, in this case, resolved the source of infection and also lowered voiding pressures. By "radical" TURP (almost complete removal of the prostate adenoma), the microabscesses that were acting as a "nest" for the microbes were eradicated [11-13]. Additionally, the TURP assisted with low-pressure conditions with limited urethral resistance [14].

In neurogenic male patients with rUTI symptoms felt to be due to chronic prostatitis, TURP may be a valuable treatment option. All predisposing factors such as foreign body, urinary tract stones, altered bladder pressures and high residual urine must first be remediated. Once these efforts fail, TURP may be a valuable treatment option.

\section{Compliance with ethical standards}

Conflict of interest The authors declare that they have no conflict of interest. 
Informed consent Written informed consent was obtained from the patient. The patient consented of the publication of the data.

Publisher's note Springer Nature remains neutral with regard to jurisdictional claims in published maps and institutional affiliations.

\section{References}

1. Konstantinidis C, Karafotias A. Urinary tract infections in neuropatients. In: Behzadi $P$, editor. Microbiology of urinary tract infections-microbial agents and predisposing factors. London, UK: IntechOpen Open Access Publisher; 2018.

2. Kennelly M, Thiruchelvam N, Averbeck MA, Konstantinidis C, Chartier-Kastler E, Trøjgaard $\mathrm{P}$, et al. Adult neurogenic lower urinary tract dysfunction and intermittent catheterisation in a community setting: risk factors model for urinary tract infections. Adv Urol. 2019;2019:2757862. https://doi.org/10.1155/2019/2757862.

3. Bonkat G, Pickard R, Bartoletti R, Bruyère F, Cai T, Geerlings SE, et al. EAU Guidelines on Urological Infections. European Association of Urology. 2019. https://uroweb.org/wp-content/uploads/ EAU-Guidelines-on-Urological-infections-2019.pdf

4. Barbalias GA, Nikiforidis G, Liatsikos E. $\alpha$-Blockers for the treatment of chronic prostatitis in combination with antibiotics. J Urol 1998;159:883-7.

5. Sobel JD. Pathogenesis of urinary tract infections. Host defenses. Infect Dis Clin North Am 1987;1:751-72.
6. Bergamin PA, Kiosoglous AJ. Surgical management of recurrent urinary tract infections: a review. Transl Androl Urol 2017;6 (Suppl 2):S153-62.

7. Decaestecker K, Oosterlinck W. Transurethral resection of the prostate in recurrent acute bacterial prostatitis. Urol Int. 2015;94:442-4.

8. American Spinal Injury Association. International standards for neurological classifications of spinal cord injury. Revised ed. Chicago, IL: American Spinal Injury Association; 2000. p. 1-23.

9. Maynard FM Jr, Bracken MB, Creasey G, Ditunno JF Jr, Donovan WH, Ducker TB, et al. International standards for neurological and functional classification of spinal cord injury. American Spinal Injury Association. Spinal Cord. 1997;35: 266-74.

10. Schoeb DS, Schlager D, Boeker M, Wetterauer U, Schoenthaler M, Herrmann TRW, et al. Surgical therapy of prostatitis: a systematic review. World J Urol 2017;35:1659-68.

11. Barnes RW, Hadley HL, O'Donoghue EP. Transurethral resection of the prostate for chronic bacterial prostatitis. Prostate 1982;3: $215-9$.

12. Smart CJ, Jenkins JD. The role of transurethral prostatectomy in chronic prostatitis. Br J Urol 1973;45:654-62.

13. Tarfusser Vvo BZ, Folmerz P. Endosurgical alternatives in the treatment of the chronic prostatitis syndrome: part II. The transurethral procedures. J Endourol. 1993; Suppl 1:S182.

14. De Nunzio C, Brassetti A, Gacci M, Finazzi Agrò E, Carini M, Presicce F, et al. Patients with prostatic inflammation undergoing transurethral prostatic resection have a larger early improvement of storage symptoms. Urology 2015;86:359-65. 